\title{
Redundancy in the perception of bilateral symmetry in dot textures
}

\author{
BILL JENKINS \\ University of Western Australia, Nedlands, Western Australia, Australia
}

\begin{abstract}
Redundancy in the perception of bilateral symmetry in dynamic dot textures was investigated using two-alternative forced-choice techniques. It was found that the symmetry information utilized by the visual system fell within a strip approximately 1 deg wide about the central axis of symmetry, irrespective of the size of the texture at the retina. Outside this strip, the symmetry information was found to be completely redundant.
\end{abstract}

The purpose of this paper is to investigate the relative contribution of different parts of a bilaterally symmetric texture to the perception of the global attribute, bilateral symmetry. "Symmetry" is commonly classified as a simple harmony of proportions, of balance, of which there are three forms: reflection, translation, and rotation. The general form, however, requires the invariance of a configuration of elements under a group of automorphic transformations (Weyl, 1952).

Mach (1886) was the first to direct attention to the importance of symmetry to our understanding of the visual process, and, as he pointed out in a footnote, "Some forty years ago, in a society of physicists and physiologists, I proposed for discussion the question why geometrically similar figures were also optically similar. I remember quite well the attitude taken with regard to this question, which was accounted not only superfluous, but even ludicrous. Nevertheless, I am now as strongly convinced as I was then that this question involves the whole problem of form vision"' (p. 109).

More recent researchers have agreed with Mach that symmetrical patterns are capable of providing information about the structure and function of the human visual system (Glass, 1969; Glass \& Perez, 1973; Glass \& Switkes, 1976; Julesz, 1971; Jenkins, Note 1). For example, Glass (1969) and Jenkins (Note 1) have argued that, in the specific case of transpositional symmetry, perceiving the uniformity of identical point-pairs is a function of the existence of orientationally selective cortical cells, similar to those described by Hubel and Wiesel (1977) in the macaque monkey visual cortex.

It has also been claimed that the perception of bi-

This research was supported by a grant from the Australian Research Grants Committee to the author. H. J. Jenkins is thanked for her constructive criticism and research assistance. The author's mailing address is: Psychology Department, University of Western Australia, Nedlands, W.A. 6009, Australia. lateral symmetry in dot textures reflects the existence of processes which reduce redundancy in the image. There have been at least two suggested methods of achieving this reduction of redundancy in the perception of bilateral symmetry.

Barlow and Reeves (1979) have suggested that the organism reconstructs an image on the basis of minimal information, since, as they state, "Undoubtedly it is not possible for a visual image to be represented completely, and any regularity such as symmetry is valuable for the very reason that it represents more of the image than an arbitrary or irregular feature" (Barlow \& Reeves, 1979, p. 793).

Barlow and Reeves point out that one of the advantages of symmetry is that it allows the image to be "described economically. For instance, if one half of an object is the mirror image of the other half, then one half need not be described at all" (p. 793).

The second means of redundancy reduction reflected by the perception of bilateral symmetry has been expressed by Julesz (1971). First, he proposes that "there is a point-by-point comparison process, based on neural anatomy, that has a symmetrical organization around the center of the fovea" (p. 131). By itself, this is not a particularly economical process, since it requires a comparison of each point with every other point about any possible axis of symmetry. However, Julesz has made a further suggestion which appears to recognize the value of redundancy reduction. "This point-by-point symmetrical representation is strongly weighted in favor of areas close to the axes of symmetry" (Julesz, 1971, p. 131).

The first of these two views suggests that redundancy reduction in the perception of bilateral symmetry is achieved by ignoring the reflected half of the symmetrical pattern; the second implies that the redundancy reduction is achieved by giving relatively greater weight to points close to the axis of symmetry, on both sides.

The aim of this paper is to investigate the second 
proposal, which may be tested empirically by examining the relative contribution of symmetric pairs of points at varying distances from the axis of bilateral symmetry in dynamic dot textures.

Dynamic dot textures were used because the elements of this type of texture have no inherent structure or meaning. It follows that any relationship perceived in the texture is independent of the elements the texture comprises. The dynamic quality of the stimulus is achieved by the rapid sequential plotting of pairs of points, of different within-point-pair separations, but with collinear midpoints. This continuous stream of point-pairs provides a texture analogous to that depicted in Figure 1, with the addition of internal Brownian motion. Stimuli constructed in this manner have been used previously to examine stereopsis (Ross \& Hogben, 1974), bilateral symmetry (Hogben, Julesz, \& Ross, 1976), and transpositional symmetry (Jenkins, Note 1 ).

The major advantage of this dynamic construction is that it reduces the probability of interference between elements, since in a 1 -sec stream, each element is physically present for only $1.5 \mu \mathrm{sec}$. Other advantages include an even distribution of elements (no clustering) and a more perceptible stimulus under a wide range of viewing conditions (see Jenkins, Note 1).

The relative contribution of symmetric pairs of points was evaluated by determining the extent of redundancy in a bilaterally symmetric texture. This was achieved first by determining the minimum amount of symmetry information required in order to perceive symmetry, and then by determining how much information beyond the minimum was utilized in the perception of symmetric textures.

This minimum was determined by replacing noise pairs with symmetric pairs within an ever-increasing region about the center of a square random noise texture, and comparing such a stimulus with an equally dense random noise texture (Experiment 1).

The extent of symmetry information utilized beyond this minimum was then determined using two different methods which evaluated the contribution of additional symmetry information to the perception of symmetry. First, symmetric pairs in a symmetric texture were replaced by noise pairs uniformly from the outer boundaries towards the central axis, and this stimulus was compared with an equally dense, completely symmetric texture (Experiment 2 ). Second, symmetric pairs in a symmetric texture were replaced by noise pairs uniformly outwards from the central axis, and this stimulus was compared with an equally dense random noise texture (Experiment 3 ).

It was considered that the absence of discrimination between the two textures of Experiment 2 would indicate that the symmetry information which was being replaced by encroaching noise was redundant information, since it was not being utilized in the discrimination process. However, once discrimination

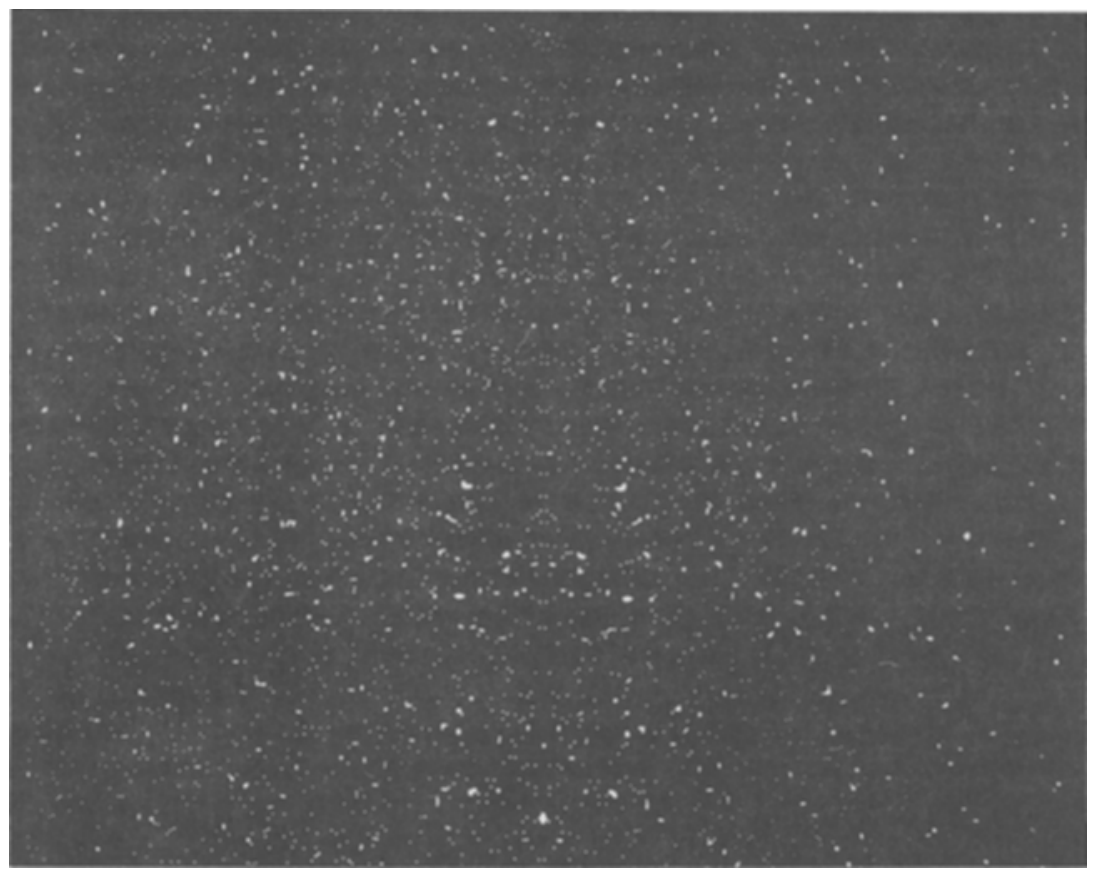

Figure 1. An illustration of a bilaterally symmetric dot texture. The dynamic textures utilized in the experiments reported here were constructed so that the appearance of the textures was similar to this static representation, but with the addition of continuous inter. nal Brownian motion. 
occurred, it was considered that the symmetry information that was being replaced was not redundant, since its absence contributed to the discrimination process.

Similarly, in Experiment 3, it was considered that discrimination between the two textures would indicate that the remaining symmetry information flanking the expanding noise was not redundant and was being utilized in the discrimination process. Once discrimination failed, however, the remaining symmetry information was not contributing to the discrimination process, and was therefore considered to be redundant.

This mapping of the relative contribution of different parts of the symmetric texture to the perception of symmetry was extended by conducting Experiments 1,2 , and 3 with three different stimulus sizes, in order to determine whether or not the contributions were dependent upon the stimulus size.

\section{GENERAL METHOD}

All experiments utilized dynamic dot textures plotted on a display oscilloscope under computer control. Subjects sat viewing the oscilloscope binocularly at a distance of $57.3 \mathrm{~cm}$. The textures were 3,5 , and $7 \mathrm{deg}$ square $(3,5$, and $7 \mathrm{~cm}$ square, respectively). The viewing distance was maintained by the use of a chin restraint for the subjects.

Stimulus luminance was $1.2 \log$ units above threshold throughout all experiments, with the oscilloscope face luminance maintained at $1 \mathrm{~cd} / \mathrm{m}^{2}$. For all experiments, the laboratory was dimly illuminated, so that the subjects did not undergo any radical change in visual sensitivity while waiting to participate in the experiments.

There was always sufficient illumination for the subjects to see the face of the oscilloscope, so that convergence and accommodation could be maintained at the appropriate distance. Each stimulus sequence was initiated by the subject, ensuring his attention to the screen at the appropriate moment.

\section{Apparatas}

The experiments were run under the control of a PDP-8/E computer, manufactured by the Digital Equipment Corporation. Interfaced to this computer was a fast display oscilloscope for presentation of the stimuli, a Hewlett-Packard 1332A oscilloscope equipped with an ultrashort P24 phosphor. The intensity of a point plotted on this type of phosphor has a single peak with a maximum spectral energy emission at $510 \mathrm{~nm}$, and the point fades to $10 \%$ of its original brightness in $1.5 \mu \mathrm{sec}$. It can therefore be considered that the effective physical duration of each point in the dynamic textures was approximately $1.5 \mu \mathrm{sec}$.

\section{Generation of Stimuli}

The dynamic textures were obtained by the rapid sequential plotting of points on an oscilloscope face within a square region, $9 \times 9 \mathrm{~cm}$. This region was a $256 \times 256$ matrix, so that the location of any point was given by one of 256 possible $x$ values and one of 256 gossible y values.

A single random point could be plotted by obtaining a random number between 0 and 256 for $x$ and, similarly, a random number for $y$. The numbers were obtained using the computer's randomnumber generator. These two coordinates, together with an intensity value, were then inserted into the appropriate locations in a computer plotting subroutine. The intensity value was held constant during all experiments. Once these values had been inserted, the next section in the program plotted the point at the specified coordinates on the oscilloscope screen.
Dynamic noise textures. Dynamic noise textures were constructed by the sequential plotting of independent random points, with the position of each new point being independent of every other point. Two random points were obtained and plotted together, with a duration of $1.5 \mu \mathrm{sec}$, and there was an interval of $121.6 \mu \mathrm{sec}$ between successive pairs of random points.

Dynamic symmetric textures. Dynamic symmetric texture fields were constructed by the sequential plotting of independent collinear point-pairs with horizontal orientation, with the position of each new point-pair being independent of every other point-pair. The position of each horizontal point-pair was determined in the same manner: the $x$ and $y$ values of a random point on either side of a vertical axis were selected independently of each other, and then the $x$ value was reflected. This new $x$ value, together with the original $y$ value, specified the coordinates of the partner point. Both points were then plotted together, with a duration of $1.5 \mu \mathrm{sec}$, and there was an interval of $121.6 \mu \mathrm{sec}$ between the plotting of successive independent point-pairs. With continuous rapid sequential plotting, the result was a texture which appeared to be bilaterally symmetric, that is, with one-half of the texture reflected about a central vertical axis.

Perceived dot density. The method of constructing dynamic textures meant that, in order to present a texture with a duration of $1 \mathrm{sec}$ at this plotting rate of two new points every $121.6 \mu \mathrm{sec}$, the computer had to plot 16,446 points $(8,223$ sequential pairs of points).

These points were distributed over the oscilloscope face $(9 \times$ $9 \mathrm{~cm}$ ) so that the distribution of points was approximately 203 points $/ \mathrm{cm}^{2}$ over the $1-\mathrm{sec}$ interval. The perceived density of the resulting texture was approximately 26 points $/ \mathrm{deg}^{2}$ of visual angle, assuming a temporal integration period of $130 \mathrm{msec}$ for this type of stimulus (Hogben \& Di Lollo, 1974; Hogben, Jules2, \& Ross, 1976).

It must be emphasized that the dynamic textures comprise a continuous train of independent pairs of points, so that at any instant, only two points are physically present on the screen. The estimate of perceived density arises from the continuous nature of this train, and not from any consideration of "frame" or frame rate (Ross \& Hogben, 1974). For all experiments and all stimulus sizes reported, the perceived density was kept constant at 26 points/ $\mathrm{deg}^{2}$ of visual angle.

\section{Experimental Comparisons}

The three experiments that were conducted required subjects to compare a standard stimulus with an experimental stimulus. Details of the particular stimuli and the specific discrimination required in each case are outlined below.

Experiment 1. The standard stimulus was a random-noise texture. The experimental stimulus was an equally dense randomnoise texture containing varying amounts of symmetric point pairs which replaced noise pairs about the central vertical axis. The independent variable was the width of the region of replacement about the axis, and five values of this variable were investigated: $.105, .175, .245, .315$, and $.385 \mathrm{deg}$ of visual angle.

The subject's task was to identify which of the two stimuli contained symmetry.

Experiment 2. The standard stimulus was a symmetric texture. The experimental stimulus was an equally dense symmetric texture containing varying amounts of noise pairs which replaced symmetric pairs uniformly from the outer boundaries towards the central vertical axis. The independent variable was the width of the remaining region of symmetry about the axis, and five values of this variable were investigated: $.3, .5, .7, .9$, and $J .1$ deg of visual angle.

The subject's task was to identify the completely symmetrical texture.

Experiment 3. The standard stimulus was a random noise texture. The experimental stimulus was an equally dense symmetric texture containing varying amounts of noise pairs which replaced symmetric pairs about the central vertical axis. The independent variable was the width of the region of replacement about the axis, 
and five values of this variable were investigated: $.3, .5, .7, .9$, and 1.1 deg of visual angle.

The subject's task was to identify which of the two stimuli contained symmetry.

The values of the independent variables in all experiments described above were established in pilot studies as those which covered the range of sensitivity for all subjects.

Each experiment was conducted with three stimulus sizes, 3, 5 , and $7 \mathrm{deg}$ square. These values were selected since this range allowed a doubling of side length and a fivefold increase in area while avoiding complications known to be associated with more eccentric parts of the retina (Jenkins, Note 1).

\section{Experimental Procedure}

The psychophysical method used was a two-alternative temporal forced-choice technique, in which each trial comprised the presentation of two textures, the standard and the experimental stimulus. Each session comprised 100 trials, 20 trials of each of five values of the independent variable presented randomly. The order of stimulus location within each trial was also random. Five sessions were completed by each subject for each experimental condition.

To initiate a trial, the subject depressed one of two hand-held push buttons, and the stimulus sequence commenced immediately. Two textures appeared on the screen sequentially, each of 1-sec duration, and were separated by a 1-sec interstimulus interval. The subject then identified the temporal location of the stimulus of interest and pressed the push button corresponding to "first" or "second" as appropriate. This decision was signaled to the computer, and display parameters were then computed for the next trial (computing time approximately $10 \mathrm{msec}$ ). Then the subject was free to initiate the next trial. There was no pressure to complete a session within a given time limit, but in practice it was found that each set of 100 trials was completed in approximately $15 \mathrm{~min}$. At the conclusion of each session, the computer printed out the results on the teletypewriter.

\section{Subjects}

The sequence of events outlined in the preceding section was conducted with three observers for each experiment. All had normal or corrected-to-normal vision.

\section{Collection of Date}

The results provided by the computer at the conclusion of each session consisted of the number of correct detections of the pointpair field for each of the five values of the independent variable, each value having been presented randomly 20 times throughout the sequence of 100 trials. The magnitudes of the five values were determined from pilot observations and were selected to maximize the range of scores from perfect discrimination to chance guessing for all three subjects.

Each session of 100 trials was repeated five times, providing 100 observations per point, which provided an adequate estimate of the psychophysical function. No more than two sessions were required of a subject in any one hour.

\section{RESULTS}

Individual results for a stimulus size of $5 \mathrm{deg}$ are described below and illustrated in Figures 2, 3, and 4. Thresholds derived from these performance curves are compared with thresholds for stimulus sizes 3 and 7 deg square in Figure 5.

The results for Experiment 1, illustrated in Figure 2, indicate that, as the width of the symmetric strip about the axis increases, detection of the symmetry improves. Taking $75 \%$ correct discrimination

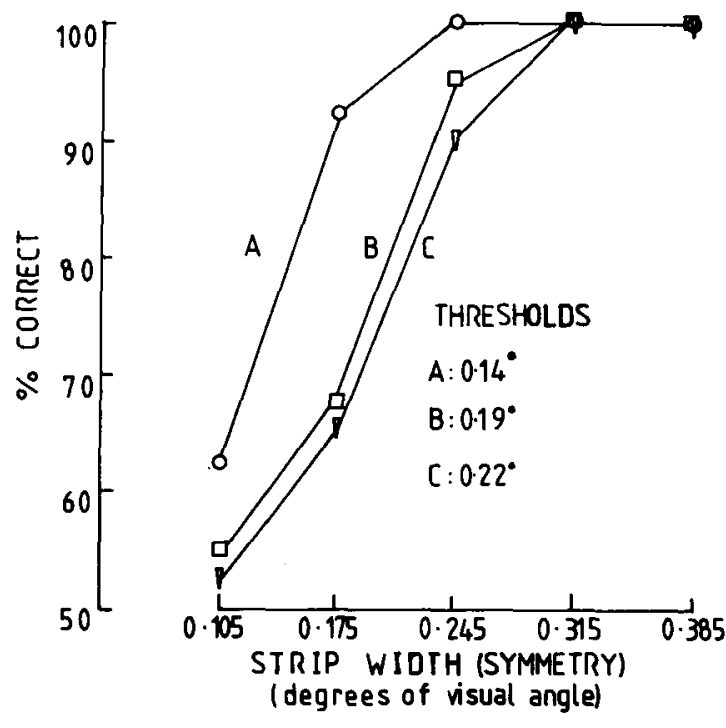

Figure 2. Experiment 1: Percent correct discrimination between a completely random noise texture and an equally dense noise texture containing an expanding strip of symmetrical pairs about the central vertical axis of the pattern. Results are shown for three subjects, and the threshold ( $75 \%$ correct discrimination) is listed for each subject. For all subjects, as the strip width increases, discrimination improves.

as the absolute threshold for the detection of symmetry, the thresholds for the three subjects, $A, B$, and $C$, are $.14, .19$, and $.22 \mathrm{deg}$ of visual angle, respectively. Subject A achieves perfect discrimination with strip widths smaller than those of Subjects B and $\mathrm{C}$, but the performance curves are qualitatively similar for all three subjects.

The results for Experiment 2 are illustrated in Figure 3, and they indicate that, as the width of the symmetric strip about the axis increases, it becomes increasingly difficult for all subjects to discriminate between the partially and the completely symmetric textures. The discrimination thresholds for Subjects $\mathrm{A}, \mathrm{B}$, and $\mathrm{C}$ are .70, .79, and .88 deg of visual angle, respectively. It is noted that there are small quantitative differences between subjects, but the three performance curves are qualitatively similar.

The results for Experiment 3 are illustrated in Figure 4, and they indicate that, as the width of the random-noise strip about the central axis increases, discrimination between the experimental stimulus containing symmetry and the equally dense randomnoise stimulus decreases. The discrimination thresholds for Subjects A, B, and C are $.80, .90$, and $.96 \mathrm{deg}$, respectively, and the performance curves are qualitatively similar for all three subjects.

The effect of stimulus size is illustrated in Figure 5, where it can be seen that, for all subjects in all experiments, the discrimination thresholds do not vary consistently as a function of stimulus size. 


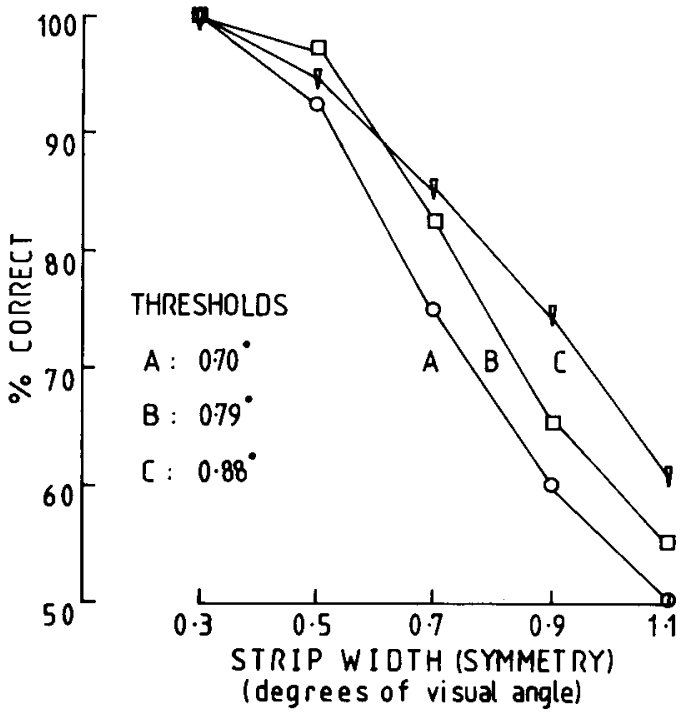

Figure 3. Experiment 2: Percent correct discrimination between a completely symmetrical texture and an equally dense symmetrical texture with noise encroaching from the outer boundaries. Results are shown for three subjects, and the threshold $(75 \%$ correct discrimination) is listed for each subject. For all subjects, as the strip width decreases, discrimination improves.

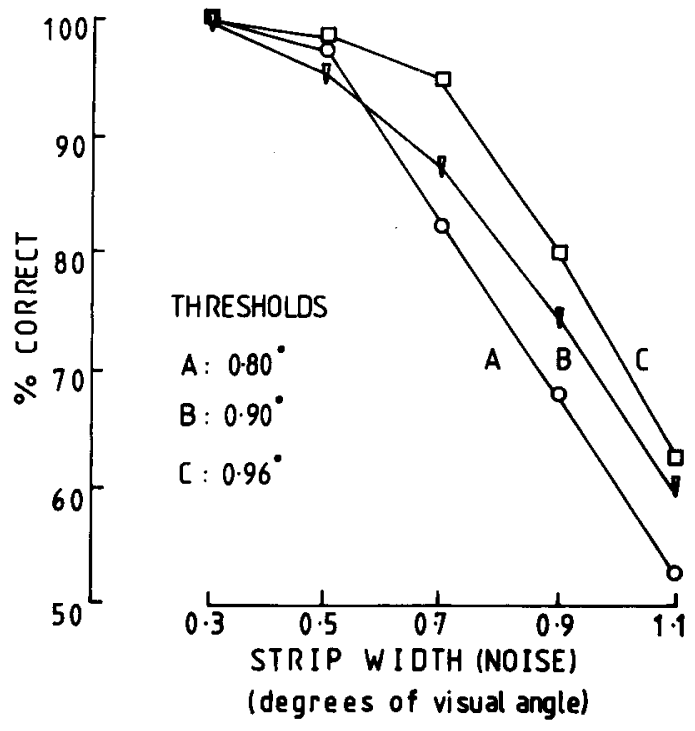

Figure 4. Experiment 3: Percent correct discrimingtion between a completely random noise texture and an equally dense symmetric texture with an expanding strip of noise about the central vertical axis. Results are shown for three subjects, and the threshold (75\% correct discrimination) is listed for each subject. For all subjects, as the strip width increases, discrimination is impaired.

\section{DISCUSSION}

The initial aim of this investigation was to determine the minimum amount of symmetry information required in order to perceive symmetry, and the results for Experiment 1 indicate that the threshold for the detection of symmetry is a strip with a width between .14 and $.22 \mathrm{deg}$, centered about a vertical axis of symmetry. Errorless detection of symmetry was achieved by all subjects with a strip width of approximately .31 deg about the central vertical axis.

The question posed immediately by these measurements is that if such a small amount of symmetry in-

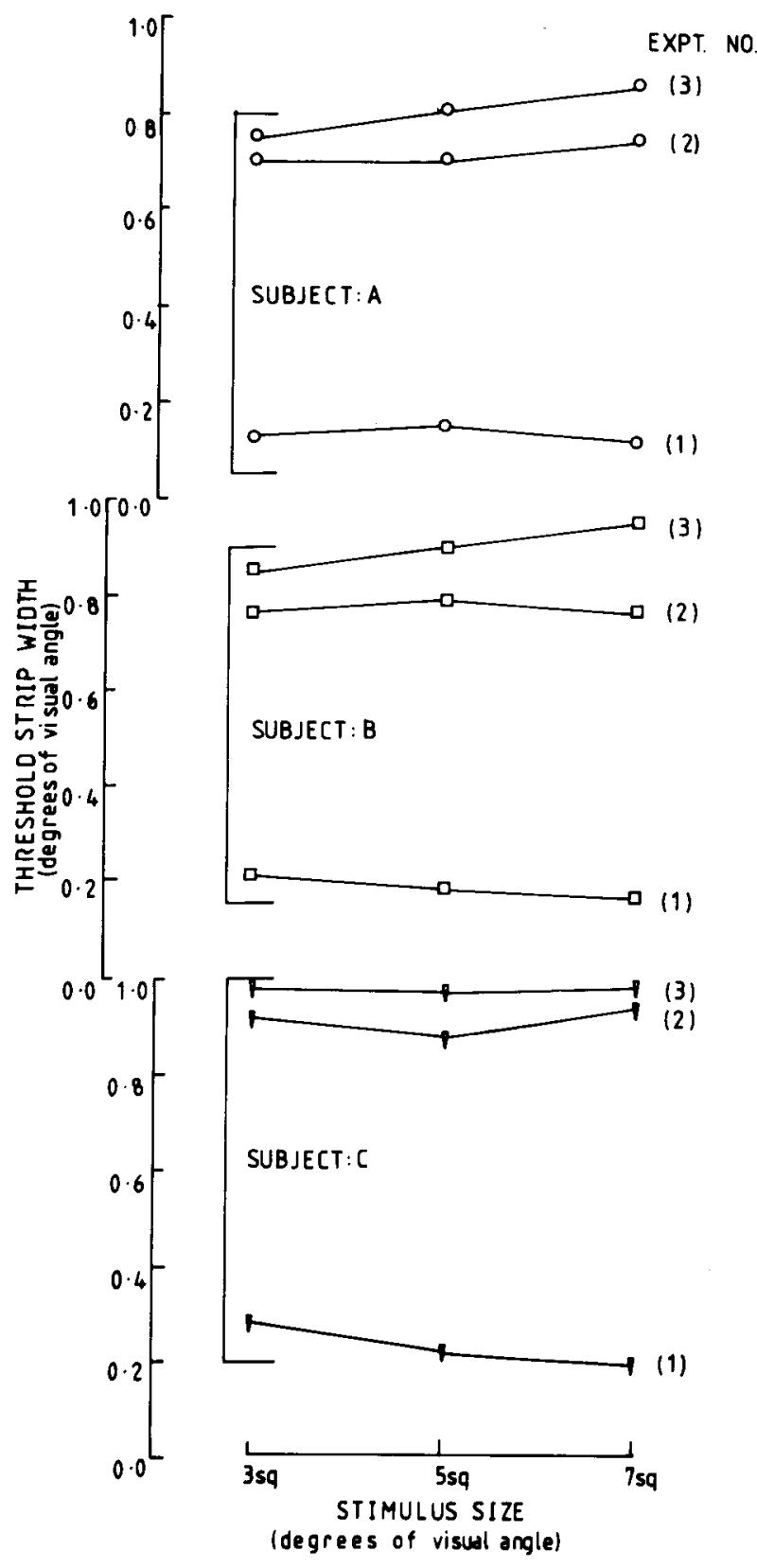

Figure 5. Threshold strip widths (symmetry or noise depending on the experiment) are shown for three subjects, for three different stimulus sizes, for Experiments 1, 2, and 3. There is no consistent effect of stimulus size on threshold. 
formation is required in order to detect symmetry, then to what extent does the visual system utilize the remaining symmetry information in perceiving symmetry in a completely symmetric texture?

This question was answered by the second and third experiments, which indicated that the symmetry information contained in a strip up to approximately $1.1 \mathrm{deg}$ of visual angle about the central axis was utilized in the detection of symmetry. Beyond this point (strip width at which chance discrimination occurred), symmetry information did not contribute to the detection of symmetry. Both Experiments 2 and 3 , using different means of measuring the relative contribution of the symmetry information, provided similar estimates.

These results confirm that not all the symmetry information available in a symmetric texture is utilized by the visual system in the perception of symmetry. Furthermore, from investigations conducted with different stimulus sizes, it appears that the nonredundant information falls within a region approximately $1.1 \mathrm{deg}$ wide, regardless of the size of the retinal area stimulated by the symmetric textures. This variation in stimulus size at the retina was achieved by systematic occlusion, with viewing distance and density held constant. The contribution of both these factors needs to be assessed independently in order to determine whether the horizontal extent of the critical region is independent of textural characteristics.

These findings support the proposal made by Julesz that, in the process of perceiving bilateral symmetry, relatively greater weight is given to those areas close to the axis of symmetry. Better definition of "areas close to the axis" is provided by the results of Experiments 2 and 3 , which show that the area is no greater than a strip approximately $1.1 \mathrm{deg}$ wide about a vertical axis.

The results of these experiments also allow a description of the weighting function suggested by Julesz. Outside the 1.1-deg strip, the symmetry information is not utilized. However, symmetry is detected with complete accuracy with a strip width of only $.31 \mathrm{deg}$ (Experiment 1). This raises questions concerning the utilization of symmetry information within the region between .31- and 1.1-deg strip width.

The results of Experiment 2 demonstrate that, as symmetry information within the region from 1.1 to $.3 \mathrm{deg}$ is replaced by noise, there is a gradual increase in discrimination between this texture and a completely symmetric texture.

Similarly, in Experiment 3, as the symmetry information disappears from the region .3 to 1.1 deg progressively outwards, there is a gradual decrease in discrimination between the texture and a completely random texture.

Together, these results suggest that there is an increasingly efficient utilization of symmetry information from the outer boundaries of the 1.1-deg strip inwards, until, at $.3 \mathrm{deg}$, the symmetry information is utilized maximally.

Therefore, it seems plausible to describe the relative contribution of the symmetry information as a continuous function which gives increasingly greater weight to symmetric pairs of points falling within the region from 1.1 to $.3 \mathrm{deg}$ about the axis of symmetry.

When a completely random dot texture is reflected about a vertical axis, the resultant bilateral symmetry is distinguished by the presence of an immediately perceptible "cluster" or "feature" which subjects readily identify as the location of the axis of symmetry because the feature appears symmetrical. It is therefore possible that the discriminations reported in Experiments 1 and 2 correspond to the detection of the presence or absence of this perceptual feature, and that the strip between 1.1 and $.3 \mathrm{deg}$ indicates some favorable, or optimal, width for the central feature. If this is so, then it is unnecessary to postulate any special mechanism, such as point-by-point comparisons, by which the visual system detects the presence of symmetry information in dot textures, since the problem "reduces" to the general case of identifying the symmetry of a feature.

However, the results of Experiment 3 suggest that point-by-point comparisons are essential to the discrimination process, since discrimination is possible in the absence of this central symmetric feature. The visual system was able to detect the symmetry information when the symmetric pairs subtended a minimum of less than $1.1 \mathrm{deg}$, and the remainder of the internal strip was filled with random noise. It should be noted that the dynamic nature of the stimulus precluded the existence of contours or boundaries between the noise and symmetric parts of the dynamic texture. Therefore the visual system is capable of detecting the symmetry information in the absence of anything resembling a feature.

Moreover, this capability has been demonstrated to have spatial limits which are independent of stimulus size, suggesting that these limitations may be structural limitations, at the retina or beyond.

In conclusion, the experiments reported in this paper have shown that (1) not all the symmetry information in a symmetric dot texture is used in the perception of symmetry, and (2) the least redundant information exists in a 1.1-deg strip about the central axis of symmetry, and this strip width is independent of stimulus size.

Several issues emerge as a result of these findings, and of particular interest is the possible means by which the visual system evaluates symmetry information in the absence of symmetric "features."

\section{REFERENCE NOTE}

1. Jenkins, B. Spatial limits to the perception of transpositional symmetry in dot textures. Paper presented to the Experimental Psychology Conference, University of Adelaide, May 1981. 


\section{REFERENCES}

Barlow, H. B., \& Reeves, B. C. The versatility and absolute efficiency of detecting mirror symmetry in random dot displays. Vision Research, 1979, 19, 783-793.

Glass, L. Moiré effects from random dots. Nature, 1969, 223, 578-580.

Glass, L., \& Perez, R. Perception of random dot interference patterns. Nature, 1973, 246, 360-362.

Glass, L., \& Switkes, E. Pattern recognition in humans: Correlations which cannot be perceived. Perception, 1976, 5, 67-72.

Hogben, J. H., Julesz, B., \& Ross, J. Short-term memory for symmetry. Vision Research, 1976, 15, 1-6,

Hogben, J. H., \& Di Lollo, V. Perceptual integration and perceptual segregation of brief visual stimuli. Vision Research, $1974,14,1059-1070$.
HUBel, D., \& WiEsel, T. N. Ferrier lecture: Functional architecture of macaque monkey visual cortex. Proceedings of the Royal Society, London, B, 1977, 198, 1-59.

Julesz, B. Foundations of cyclopean perception. Chicago: Chicago University Press, 1971.

MACH, E. The analysis of sensations and the relation of the physical to the psychical. New York: Dover, 1959. (1st German ed. published, 1886.)

Ross, J., \& HogbeN, J. H. Short-term memory in stereopsis. Vision Research, 1974, 14, 1195-1201.

Weyl, H. Symmetry. Princeton, N.J: Princeton University Press, 1952.

(Manuscript received February 3, 1982; revision accepted for publication April 21, 1982.) 